Artículo científico

Volumen 31(1):13-29. Enero-abril, 2020

e-ISSN 2215-3608, doi:10.15517/am.v31i1.36887

http://www.revistas.ucr.ac.cr/index.php/agromeso

\title{
Cadena de valor en la red de tomate de árbol (Solanum betaceum) en Ecuador $^{1}$
}

\section{The value chain of tree tomato (Solanum betaceum) network in Ecuador}

\author{
Carlos Moreno-Miranda ${ }^{2}$, José Isaac Molina ${ }^{3}$, Jacqueline Ortiz ${ }^{3}$, Carla Peñafiel ${ }^{3}$, Raúl Moreno ${ }^{4}$
}

1 Recepción: 10 de abril, 2019. Aceptación: 5 de setiembre, 2019. Este trabajo fue resultado de una investigación realizada durante el 2016 y 2017 y recibió el apoyo de la Facultad de Ciencia e Ingeniería en Alimentos de la Universidad Técnica de Ambato, Ecuador.

2 Wageningen University \& Research, Agricultural Economics and Rural Policy Department, Campus Hollandseweg 1, 6706 KN Wageningen. Wageningen, The Netherlands. carlos.morenomiranda@wur.nl (autor para correspondencia; http://orcid.org/0000-0002-8378-7325).

3 Universidad Técnica de Ambato, Facultad de Ciencia e Ingeniería en Alimentos, Campus Huachi, Av. de Los Chasquis, Ambato, Ecuador. ji.molina@uta.edu.ec,jortizes@uta.edu.ec,ca.penafiel@uta.edu.ec

4 Universidad de Barcelona, Facultad de Ciencias de la Alimentación, Campus Diagonal, Av. de Joan XXIII, 27-31, Barcelona, España. rmorenm07@alumnes.ub.edu

\section{Resumen}

Introducción. El sector de las frutas y hortalizas en Ecuador ha mostrado un desempeño prometedor en mercados de tipo doméstico e internacional. Este sector ha enfrentado problemas de carácter social, económico y productivo en su estructura y articulaciones, que hasta el momento requieren de intervención. Objetivo. El objetivo de este estudio fue analizar de manera descriptiva la estructura de la red agroalimentaria del tomate de árbol. Materiales y métodos. El estudio se realizó en las provincias de Cotopaxi, Tungurahua y Chimborazo, Ecuador, durante el periodo comprendido entre el segundo cuatrimestre de 2016 al último del 2017. En el estudio se aplicó una metodología sistémica encaminada a la identificación y caracterización de etapas, agentes y actividades (primarias y de soporte); y el análisis de los mecanismos de gobernanza de la red. Resultados. El presente estudio mostró las principales características sociales y productivas, un dimensionamiento horizontal y vertical; y la propuesta de estrategias enfocadas en la mejora de su desempeño productivo. Conclusiones. La inclusión de mujeres, el sentido de asociatividad de procesadores y distribuidoras, y la formación de productores en poscosecha, y buenas prácticas agrícolas, mejorarían significativamente el desempeño económico de la red, y además incentivarían una mayor participación de los agentes involucrados. La red requiere aumentar el área asignada al cultivo de tomate de árbol, y la aplicación de sistemas de producción bajo invernadero que incrementen los rendimientos.

Palabras claves: coordinación, horticultura, unidad de producción, mercado, estrategia.

\begin{abstract}
Introduction. The fruit and vegetable sector in Ecuador has shown promising performance in domestic and international markets. Likewise, this sector has faced problems of a social, economic and productive nature in its structure and articulations, which until now require intervention. Objective. The objective of this study was to
\end{abstract}


analyze in a descriptive way the structure of the tree tomato agro-food network. Materials and methods. This study was carried out in the provinces of Cotopaxi, Tungurahua, and Chimborazo, Ecuador, during the period between the second quarter of 2016 and the end of 2017. The study applied a systemic methodology aimed at the identification and characterization of stages, agents and activities (primary and support), and the analysis of network governance mechanisms. Results. The present study showed the main social and productive characteristics, horizontal and vertical sizing; and the proposal of strategies focused on improving their productive performance. Conclusions. The inclusion of women, the sense of associativity of processors and distributors, and the training of producers in topics related to post-harvest, and good agricultural practices, would significantly improve the economic performance of the network, and also encourage greater participation of the agents involved. The network requires increasing the area allocated to tree tomato cultivation, and the application of greenhouse production systems that increase yields.

Keywords: coordination, horticulture, unit of production, market, strategy.

\section{Introducción}

Durante el período 2005 - 2015 los países andinos como Perú, Chile, Ecuador y Colombia presentaron una tendencia creciente en la producción de frutas y vegetales (Olaizola et al., 2006; Brown y Torres, 2012; Ordoñez, 2012; Gayá y Michalczewsky, 2014). Este crecimiento fue evidenciado por el aumento de exportaciones hortofrutícolas en esos países de toda América Latina, cuyo promedio anual fue de 4,2 \% (FAO, 2011; CEPAL et al., 2015). Las cadenas productivas de frutas y vegetales de países centroamericanos experimentaron fuertes pérdidas en sus etapas y subsistemas de producción, debido a sequías, plagas, enfermedades y condiciones climáticas (Blandon et al., 2009; Reardon y Flores, 2006; Urquilla, 2012). Este acontecimiento en países centroamericanos jugó un papel importante, porque incentivó la producción hortofrutícola en Sudamérica (Sánchez, 2017; Giacobone et al., 2018). Sin embargo, fue inevitable una caída general del dinamismo de exportaciones agroalimentarias de América Latina, debido a la ausencia de planes de contingencia que contrarresten los factores biofísicos mencionados (FAO, 2011; CEPAL et al., 2015).

El sector agropecuario de los países latinoamericanos aún presenta desventajas relacionadas con la eficiencia, el rendimiento, tecnología y el manejo poscosecha (Pérez, 2004; Acosta 2006; Gil-Méndez, 2015; Rodríguez, 2015). Al mismo tiempo, los aspectos que elevan su competitividad son los bajos costos de producción en relación a países de otros continentes, la diversidad de clima, suelo y genética vegetal (Vergara et al., 2014; López, 2015). Sin embargo, respuestas integrales que vinculen aspectos sociales, ambientales, económicos e institucionales, aún se encuentran en debate (Miranda, 2011; Scott, 2014; Carpio, 2018). Por lo tanto, los actores de las cadenas productivas deben intervenir a través de la implementación de tecnología y sistemas de gestión sostenibles (Orjuela et al., 2008).

El sector hortofrutícola en Ecuador ha mostrado un crecimiento al aportar el $16 \%$ al PIB agrícola sin considerar la producción de papas y banano (FAO, sf; Glas et al., 2015; MAGAP, 2016). En el caso de explotaciones vegetales, las unidades de producción son relativamente pequeñas con superficies menores a las 10 ha y una alta diversificación de productos. Asimismo, se caracterizan por incidir frecuentemente en altos costos transaccionales, debido a la falta de planificación en logística y mejoramiento de infraestructura que coordinen las actividades (Barrera-Mosquera et al., 2010; Aguilar, 2017; Páez, 2018). En cambio, las explotaciones frutales presentan superficies que superan las 10 ha y con un menor grado de diversificación de productos. Adicionalmente, estas se caracterizan por sus mecanismos eficientes en la coordinación de la logística de cosecha, clasificación y otros procedimientos que demandan los mercados locales y extranjeros (Demenus y Crespo, 2011; Herforth et al., 2015). 
La horticultura es una actividad económica viable y emergente para las familias campesinas ecuatorianas, gracias a las condiciones agronómicas rurales (Melo y Hollander, 2013; Rimisp, 2019). El caso de la producción de tomate de árbol es un ejemplo de actividad agrícola emergente de la zona interandina (Wendt e Izquierdo, 2003; Campuzano, 2014). La asignación de recursos agrícolas en los sistemas de producción y el financiamiento mediante programas públicos, han fortalecido progresivamente su desempeño productivo (Padilla y García, 2018; Quiroso et al., 2008). Sin embargo, la persistencia de dificultades socio-productivas requieren soluciones que apuntalen el dinamismo en mercados locales y globales (Cadilhon et al., 2006; Craig et al., 2011; Coyle et al., 2013; Barbier, 2016). También, es necesario comprender los mecanismos que gobiernan y coordinan una cadena con la finalidad de canalizar eficientemente recursos financieros, materiales y humanos (Gereffi, 1994). Por tanto, son necesarios estudios sobre la configuración y aspectos socio-productivos para identificar debilidades, comprender la gobernabilidad y establecer soluciones estratégicas y sostenibles.

El tomate de árbol (Solanum betaceum), también llamado Tamarillo, es originario de Sudamérica. Los principales países productores son Ecuador y Colombia. La planta es semi leñosa de 2 a $3 \mathrm{~m}$ de altura (Castro, 2014). La fruta es una baya elíptica de forma ovoidal, mide entre 5 y $10 \mathrm{de} \mathrm{cm}$ de largo, y de 8 a $5 \mathrm{~cm}$ de diámetro (Flores, 2007). Además, la fruta está cubierta por una cáscara gruesa y amarga en tonos rojos, naranjas y amarillos, según el ecotipo (Feican, 2016); en estado de madurez, pesa entre 80 y 90 g. El ciclo de producción inicia a los ocho y nueve meses después del trasplante en campo (León et al., 2004). La vida comercial de la planta es de ocho años (Castro, 2014). El fruto es rico en potasio, fibra y tiene vitamina A, B, C y K. Además, los principales beneficios del consumo de tomate de árbol son la reducción del colesterol y el aporte de fibra que favorece el tracto intestinal (Arboleda et al., 2013). Por tanto, este producto agrícola presenta características que motivan su estudio e investigación.

En la región Sierra Ecuatoriana, durante el periodo 2015-2017, el área de cultivo de tomate de árbol se incrementó en un $70 \%$, pasando de 4500 a 7600 ha (FAO, 2018). Actualmente, las provincias más sobresalientes son Imbabura, Tungurahua y Pichincha (MAGAP, 2016). Según el Ministerio de Agricultura y Ganadería (MAG, 2018), el tomate de árbol se ubica en el décimo puesto de los cultivos frutícolas interandinos en términos de rendimiento, y en el puesto décimo quinto en términos de área cultivada. El área cultivada y cosechada, así como el rendimiento por hectárea, presentan una tendencia de crecimiento en los últimos años (Viteri et al., 2010; Feican, 2016).

El Banco Central del Ecuador informó que durante el periodo 2010-2014 hubo un incremento en las exportaciones, pasando de 20500 a 27000 t con respecto al periodo 2005-2009. Actualmente, se calcula que existe una producción anual de 39725 t de tomate de árbol. Al menos 30500 t son aprovechadas por la agroindustria y exportadoras, mientras que $8600 \mathrm{t}$ son comercializadas en estado fresco a nivel local y regional (PROECUADOR, 2016). Sin embargo, el conocimiento de su dinámica económica y desempeño socioproductivo aún presenta brechas que deben ser cubiertas. El objetivo de este estudio fue analizar de manera descriptiva la estructura de la red agroalimentaria del tomate de árbol.

\section{Materiales y métodos}

\section{Área de estudio}

El estudio de la estructura de la red agroalimentaria del tomate de árbol (Solanum betaceum), se realizó en Ecuador, en la zona interandina comprendida por las provincias de Cotopaxi, Tungurahua y Chimborazo (extensión aproximada de $59810 \mathrm{~km}^{2}$ ) a partir del segundo cuatrimestre del año 2016 hasta el último cuatrimestre del 2017. Esta zona geográfica se localiza a $0^{\circ} 42^{\prime}$ latitud sur y $80^{\circ} 00^{\prime}$ longitud oeste, con una altitud promedio de 3500 $\mathrm{msnm}$, y una temperatura promedio que varía entre 15 y $25^{\circ} \mathrm{C}$ (Figura 1). La cadena de tomate de árbol ha recibido 


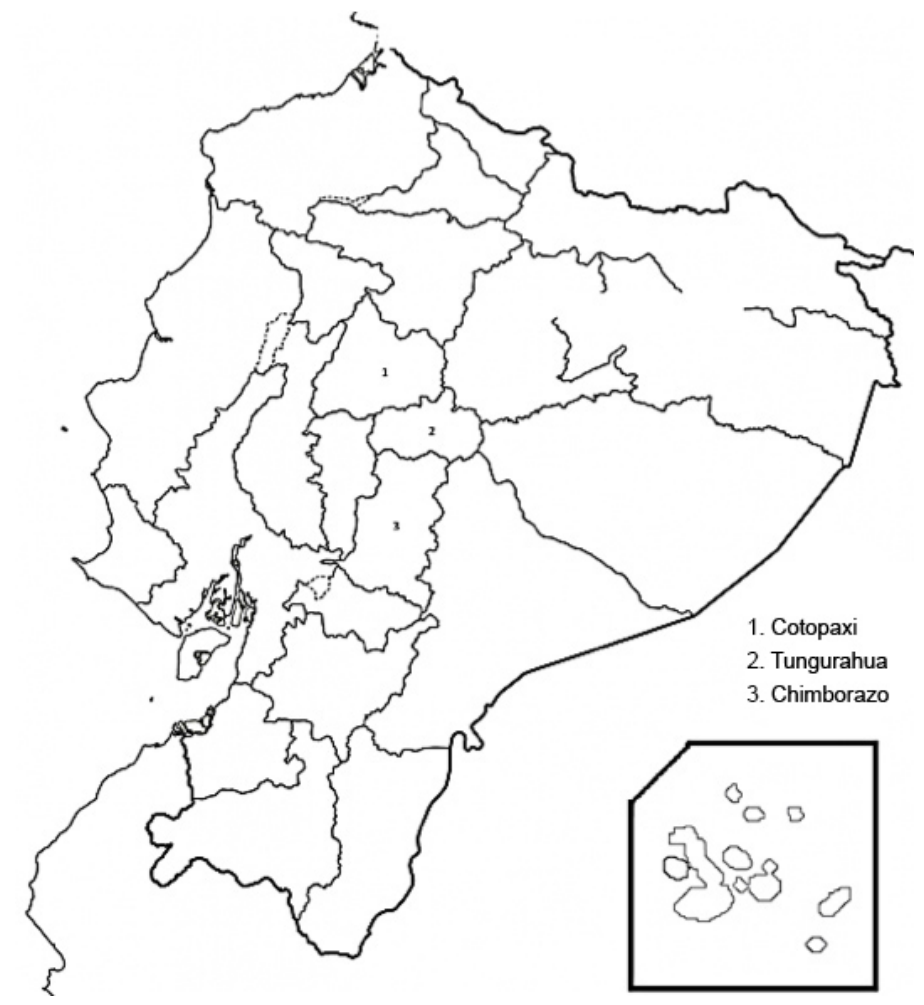

Figura 1. Mapa del territorio ecuatoriano y zona comprendida por las provincias Cotopaxi, Tungurahua y Chimborazo, donde se efectuó el estudio de la estructura de la red agroalimentaria del tomate de árbol (Solanum betaceum). Ecuador. 2016-2017.

Figure 1. Map of the Ecuadorian territory and area comprising the provinces of Cotopaxi, Tungurahua, and Chimborazo, where the study of the structure of the agro-food network of the tree tomatoes (Solanum betaceum) was carried out. Ecuador. 2016-2017.

atención de diferentes sectores, debido a su estructura agrícola, la desigualdad en el agua de riego, la pérdida de páramos y la inestabilidad de los precios del mercado.

\section{Descripción de la metodología}

La metodología se encaminó al análisis de aspectos socioeconómicos y de la cadena de valor: producción hasta uso final. Además, se destacó los mecanismos de gobernanza que influyen sobre la cadena, como se explica a continuación:

1. Mapeo de la cadena alimentaria. Para ello se empleó el esquema de la cadena de valor desarrollado por Hawkes y Ruel (2006) para determinar los grupos de actores y las funciones físicas y de coordinación que cumple cada uno. Asimismo, se identificó los flujos de menor y mayor importancia, es decir, la ruta de recorrido del tomate de árbol y los flujos de recursos de apoyo.

2. Para la identificación de actores de la cadena de valor, se utilizó la información del último censo (2015) realizado por el Ministerio de Agricultura y Ganadería (MAG), y el registro de PYME y grandes empresas presentadas por el Ministerio de Industrias y Productividad (MIPRO). 
3. La revisión de datos secundarios se complementó haciendo una serie de encuestas y entrevistas, para lo cual se ejecutó un taller con actores interesados como productores, emprendedores, expertos del Ministerio de Agricultura y Ganadería y Ministerio de la Producción; con lo que se determinó el número y ubicación de los participantes a encuestar. También, se seleccionaron las variables de índole socio-económico y productiva, en consenso, de una lista predeterminada. A partir de la información recabada en el taller, se construyó la encuesta y se validó mediante la prueba Alfa de Cronbach. Los coeficientes obtenidos en la prueba fueron mayores a 0,75, confirmando la fiabilidad del instrumento.

4. Para la estimación del tamaño de la muestra de los productores se utilizó la variable continua "número de productores registrados por MAG" dentro de la localidad mencionada. Se aplicó la fórmula de Sukhatme (1946) con un nivel de confianza del $95 \%$. La muestra de productores estuvo compuesta por 48, 75 y 57 productores de Cotopaxi, Tungurahua y Chimborazo, respectivamente. La construcción de la muestra estuvo acorde al número de productores registrados por el MAG. También, se utilizó un informe publicado por el Ministerio de Industrias y Productividad (MIPRO), que incluye a las empresas vinculadas a la cadena tomate de árbol para analizar la etapa de postproducción (MIPRO, 2018).

4. Para el análisis descriptivo cuantitativo se empleó un enfoque empírico-analítico, con lo que se especificó y delimitó el comportamiento de las variables bajo estudio. Para el análisis cualitativo se construyeron categorías con base en los resultados de las encuestas. Estas categorías fueron empleadas posteriormente para la comparación con datos de autores externos. Se empleó histogramas y gráficos de barra para la presentación de datos.

5. En la identificación de la gobernanza se aplicaron las herramientas propuestas por Frederick y Gereffi (2009), las cuales reconocen eslabones que dominan la dinámica de la cadena, identifican el liderazgo de actores focales, así como el nivel de valor agregado que se genera a través de las distintas etapas. Finalmente, se identificaron los eslabones con los cuales se podría contar para un escalamiento económico mediante estrategias.

\section{Resultados}

\section{Mapeo de la cadena alimentaria}

La cadena funciona con base en actividades de soporte en su primera etapa. Estas actividades son llevadas a cabo por agentes como empresas productoras estatales y privadas que suministran insumos como semillas, maquinaria, fertilizantes, entre otros bienes. De estas actividades, se considera crucial la actividad de soporte financiero, debido a los actores involucrados e impacto en el crecimiento de una cadena. Las cooperativas de ahorro y crédito, y el banco estatal son quienes financian las diferentes actividades a lo largo de la red productiva. Las actividades primarias de la cadena inician en la etapa de producción donde pequeños productores y empresas agrícolas se encargan de las plantaciones y actividades agronómicas. Posteriormente, los flujos de mayor importancia (tomate de árbol) atraviesan los diferentes eslabones hasta llegar al consumidor final en diversos formatos que serán explicados más adelante (Figura 2).

\section{Identificación de actores de la cadena de valor}

De acuerdo con la información proporcionada por el MAG (Cuadro 1), se identificó que para la etapa de producción de tomate de árbol de la zona comprendida bajo estudio se cuenta con el 1,5\% de productores del sector hortofrutícola. A la vez, la provincia de Tungurahua se destaca por poseer el mayor número de hectáreas para la producción de tomate de árbol.

En la etapa de poscosecha, se identificaron 32 empresas registradas en el MIPRO. El precio de compra de tomate de árbol al por mayor de las empresas era variable entre USD \$ 0,75 a \$ 0,95 por kg. Además, se observó 


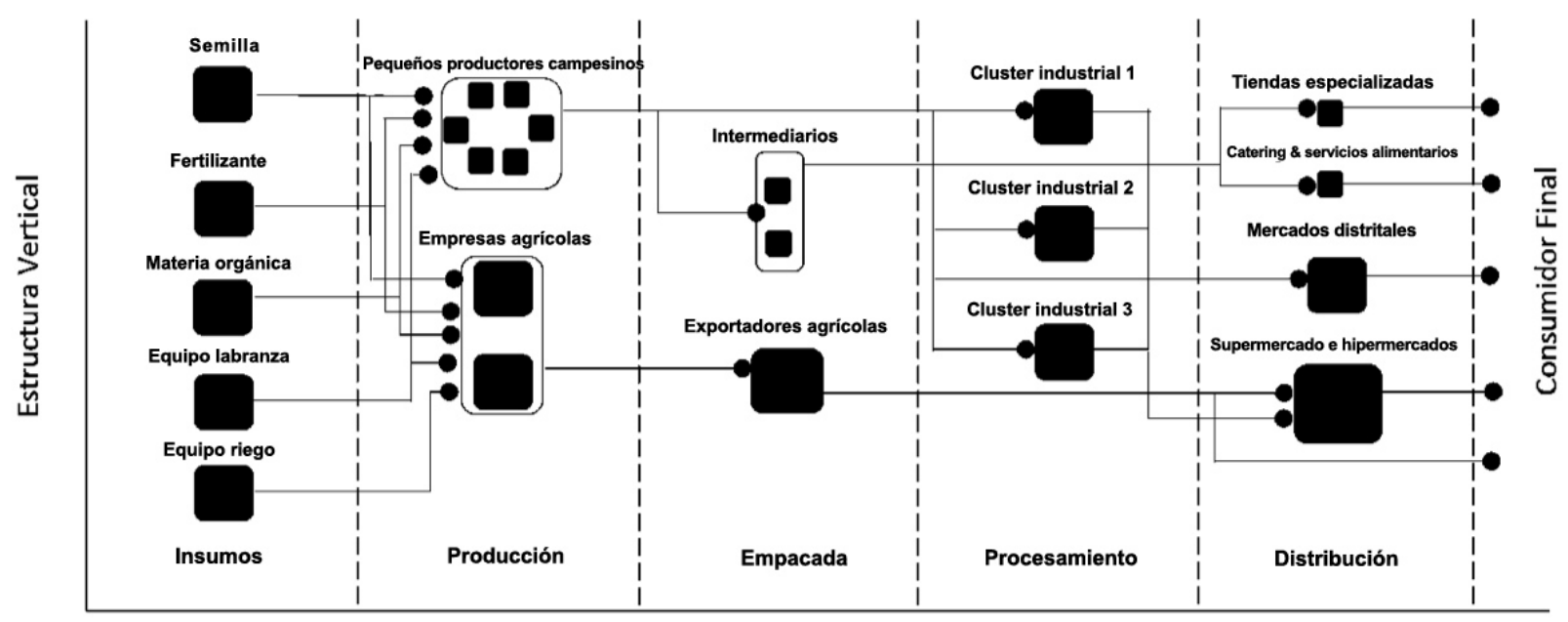

Estructura Horizontal

Figura 2. Agentes de la red productiva del tomate de árbol (Solanum betaceum), en Ecuador, en la zona Interandina comprendida por las provincias de Cotopaxi, Tungurahua y Chimborazo (extensión aproximada de $59810 \mathrm{~km}^{2}$ ). 2017.

Figure 2. Agents of the productive network of the tree tomato (Solanum betaceum), in Ecuador, in the Inter-Andean zone comprising the provinces of Cotopaxi, Tungurahua, and Chimborazo (approximate extension of 59,810 km²). 2017.

Cuadro 1. Número de productores y superficie de producción de tomate de árbol (Solanum betaceum) en la zona centro de Ecuador. 2016.

Table 1. Number of producers and production area of tree tomato (Solanum betaceum) in central Ecuador. 2016.

\begin{tabular}{ccc}
\hline Provincia & $\begin{array}{c}\text { Número de productores de tomate de árbol } \\
\text { registrados por el MAGAP }\end{array}$ & $\begin{array}{c}\text { Superficie (ha) de producción de tomate de } \\
\text { árbol registradas por el MAGAP }\end{array}$ \\
\hline Cotopaxi & 182 & 382,3 \\
Tungurahua & 250 & 485,2 \\
Chimborazo & 210 & 371,1 \\
\hline
\end{tabular}

Fuente: MAGAP (2016) / Source: MAGAP (2016).

que el 78,3\% de ellas incluían tomate de árbol en su cartera de negocios; y solo el 21,7\% transformaba el tomate de árbol en productos como jugos, pulpa, zumo, almíbares y deshidratado. El $39 \%$ de las empresas operaban exclusivamente en el mercado doméstico, y solo el 5,0 \% presentaron actividad en mercados internacionales. También, el $56 \%$ participaba en el mercado local y había tenido algún tipo de experiencia exportando (Figura 3). Por tanto, el mercado de tomate de árbol se mira en proceso de expansión tanto a nivel de oferta como de demanda.

Una debilidad de la cadena evidenciada, fue el bajo porcentaje de transformadores de tomate de árbol (21,7 $\%)$. También, se determinó que el 56,0 \% de las empresas transformadoras presentaron interés en los formatos convencionales de transformación (pulpa, conserva y jaleas) y no en alternos como suplementos. Con respecto a los distribuidores registrados, se identificaron tres corporaciones líderes del mercado minorista de tomate de árbol en estado fresco y procesado; mientras que los distribuidores restantes (diez identificados) eran empresas medianas de actividad comercial mayorista. 


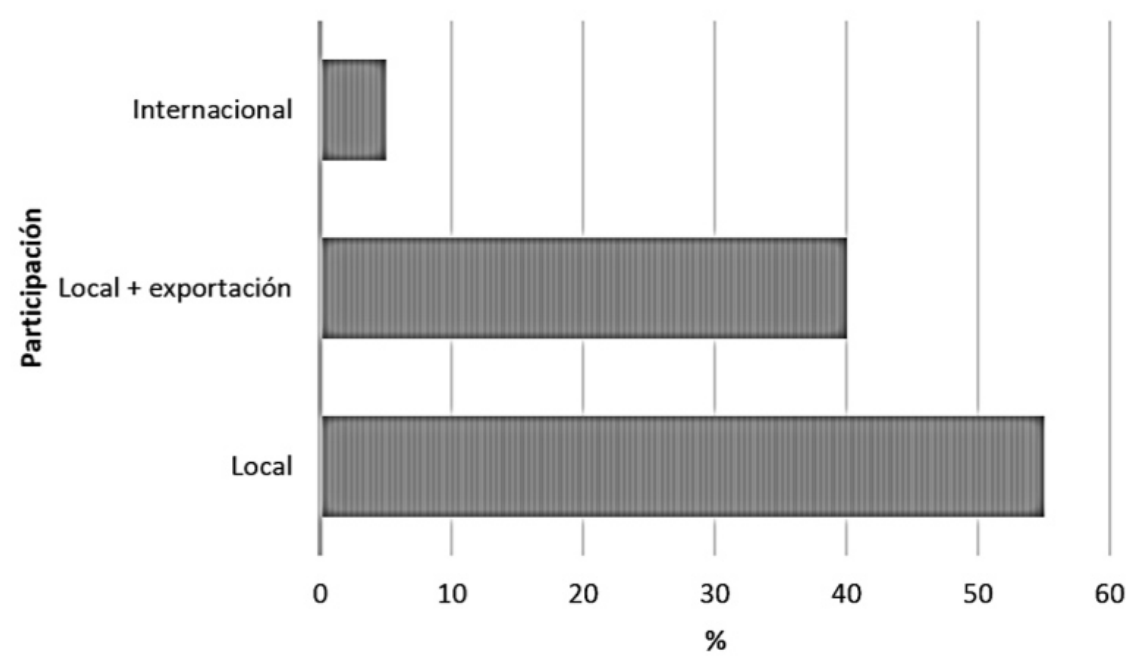

Figura 3. Participación de empresas privadas en el mercado de tomate de árbol (Solanum betaceum) en la zona centro de Ecuador. 2017.

Figure 3. Participation of private companies in the tree tomato (Solanum betaceum) market in the central area of Ecuador. 2017.

\section{Análisis descriptivo}

\section{Producción}

\section{Hallazgos socioeconómicos}

Esta etapa estuvo integrada con productores entre los 30 y 50 años, en su mayoría varones $(56,0 \%)$, pero la inclusión de mujeres se ha incrementado en un 5,0 \% en los últimos tres años gracias al aumento de oportunidades de mercado. La formación académica en su mayoría es de tipo secundaria y superior, en un 40 y $26 \%$, respectivamente. Un $28 \%$ de los productores eran miembros de asociaciones y un 39,0\% eran socios de cooperativas. La principal fuente de financiamiento ha sido el capital propio (Figura 4), pudiendo desacelerar el crecimiento económico de los productores.

\section{Hallazgos de la producción}

Se observó que un $45 \%$ poseía tierras propias, $29 \%$ de los encuestados laboraba en sociedad y un $26 \%$ arrendaba tierras. El $55 \%$ de los encuestados poseía una extensión de tierra mayor a 3 ha, y el área de cultivo para tomate de árbol iba entre 1,25 a 2,50 ha en promedio. A la vez, el $56 \%$ aplicaba técnicas de cultivo a campo abierto. El genotipo mayormente cultivado era el gigante anaranjado, seguido del genotipo morado neozelandés y en menor proporción el morado gigante (46\%, $35 \%$ y $19 \%$, respectivamente).

Durante el establecimiento del cultivo el control fitosanitario representó el mayor costo; mientras que la actividad de cosecha representó el mayor costo en el mantenimiento de la plantación (Cuadro 2). Según los encuestados, USD $\$ 0,85 \mathrm{~kg}^{-1}$ de fruta era el costo estimado, y una hectárea de cultivo representaba una TIR de al menos un $18 \%$. Al comparar esta información con datos de cultivos frutícolas de la zona, según Pérez (2011), USD \$1,07 era el costo que representaba la producción de un $\mathrm{kg}$ de mora, y una ha de cultivo tenía una rentabilidad 


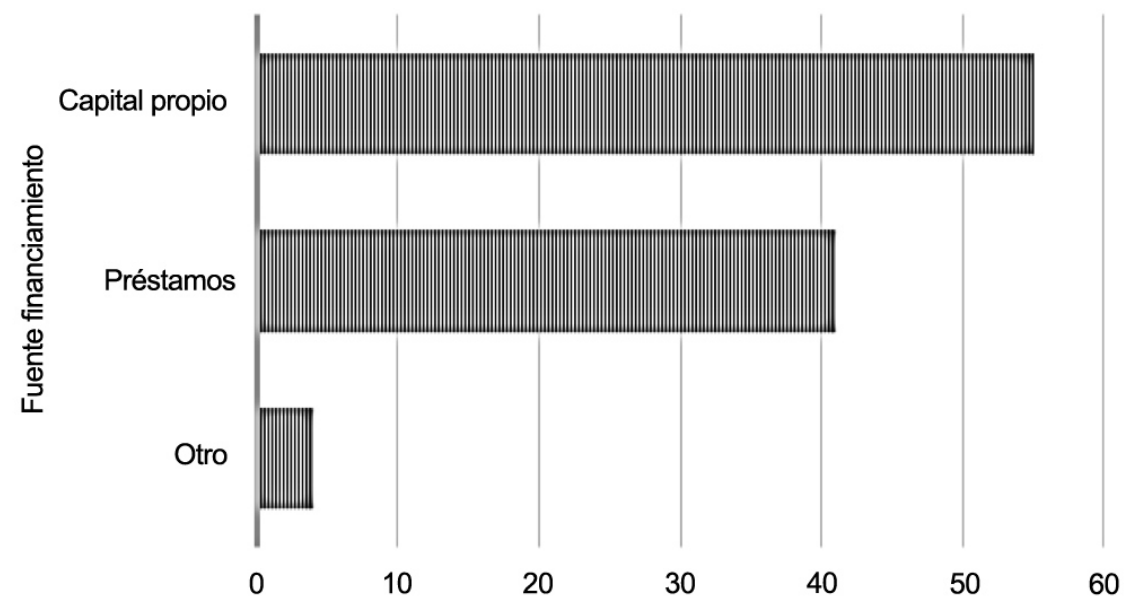

Figura 4. Fuentes de financiamiento de los productores de tomate de árbol (Solanum betaceum) en la zona centro de Ecuador. 2017.

Figure 4. Funding sources of the tree tomato (Solanum betaceum) producers in Central Ecuador. 2017.

Cuadro 2. Costos de producción de tomate de árbol (Solanum betaceum), en la zona centro de Ecuador. 2017.

Table 2. Production costs of tree tomato (Solanum betaceum), in Central Ecuador. 2017.

\begin{tabular}{|c|c|c|}
\hline Actividad & Establecimiento (\%) & Mantenimiento (\%) \\
\hline Preparación el terreno & 7 & 1 \\
\hline Siembra & 14 & 2 \\
\hline Fertilización & 24 & 5 \\
\hline Control fitosanitario & 42 & 27 \\
\hline Labores culturales & 11 & 16 \\
\hline Cosecha & 2 & 49 \\
\hline
\end{tabular}

promedio de $21 \%$. Con base en el reporte de Gunsha (2016), USD \$ 0,93 era el costo de producción de un kg de fresa, y una ha de cultivo tenía una TIR del $15 \%$.

El rendimiento promedio de producción de tomate de árbol en la zona bajo estudio fue de 15,2 $\mathrm{t} \mathrm{ha}^{-1}$. También, según expertos de entidades públicas ecuatorianas, el rendimiento de tomate de árbol para Ecuador fue de 15,3 tha-1 y para Colombia de 15,8 tha-1. Es importante mencionar que solo un $33 \%$ de los productores aplicaba técnicas de poscosecha y que consistían en: i) distribución de la cosecha en contenedores plásticos de $15 \mathrm{~kg}$ de capacidad para su transporte a bodegas o centros de acopio, y ii) selección de la fruta por tamaño (calibre) y estado de madurez. El porcentaje de quienes aplicaban técnicas de poscosecha era relativamente bajo (menos del $30 \%$ ), debido a varios factores como falta de conocimiento técnico, y escasa intervención por parte del sector académico. 


\section{Poscosecha: procesadores}

\section{Hallazgos socioeconómicos}

De las empresas registradas se identificó que más del $70 \%$ correspondían a Mipymes, y hay grandes empresas participando en la cadena de valor de tomate de árbol (Figura 5). El $64 \%$ de las empresas registradas eran miembros de asociaciones y un $36 \%$ eran socios de cooperativas.

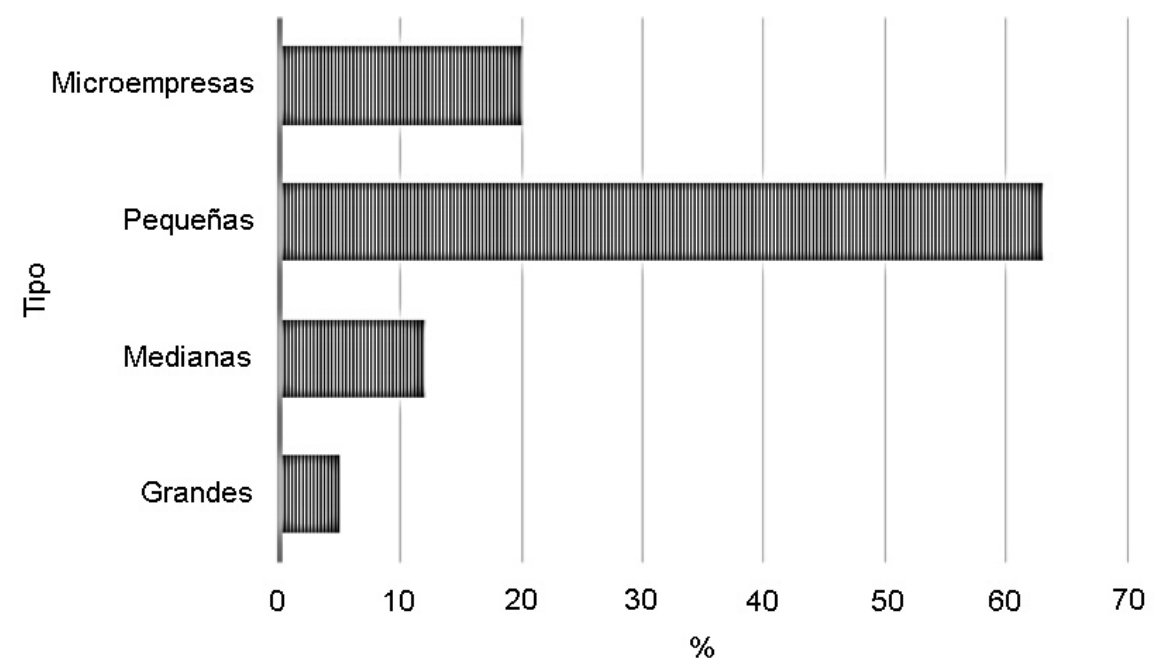

Figura 5. Tipos de empresas participantes en la cadena de valor del tomate de árbol (Solanum betaceum) en la zona centro de Ecuador. 2017.

Figure 5. Types of companies participating in the tree tomato (Solanum betaceum) value chain in central Ecuador. 2017.

Con respecto al financiamiento un $37 \%$ de los procesadores operaba mayormente con recursos propios y préstamos de entidades financieras. El $41 \%$ financia las actividades de transformación con la ayuda de préstamos y acciones en la bolsa. Se observó una relevante participación del capital de terceros como cooperativas de ahorro y crédito, entidades bancarias públicas y privadas, e inversionistas (Figura 6).

\section{Hallazgos en la comercialización}

El $57 \%$ de las empresas no ejecutaba la transacción con los proveedores cuando la fruta presentaba problemas por daños mecánicos, bajo calibre, daños por insectos, presencia de moho entre otras razones. Este hecho es observado por las empresas como riesgo en la calidad final del producto procesado. Se estableció que un $43 \%$ de los procesadores comercializaban tomate de árbol en segunda y cuarta gama, y un $57 \%$ en primera, tercera y quinta gamma. Adicionalmente, se observó que los procesadores se regían con controles sanitarios minuciosos ejecutados por la Agencia Nacional de Regulación, Control y Vigilancia Sanitaria (ARCSA). 


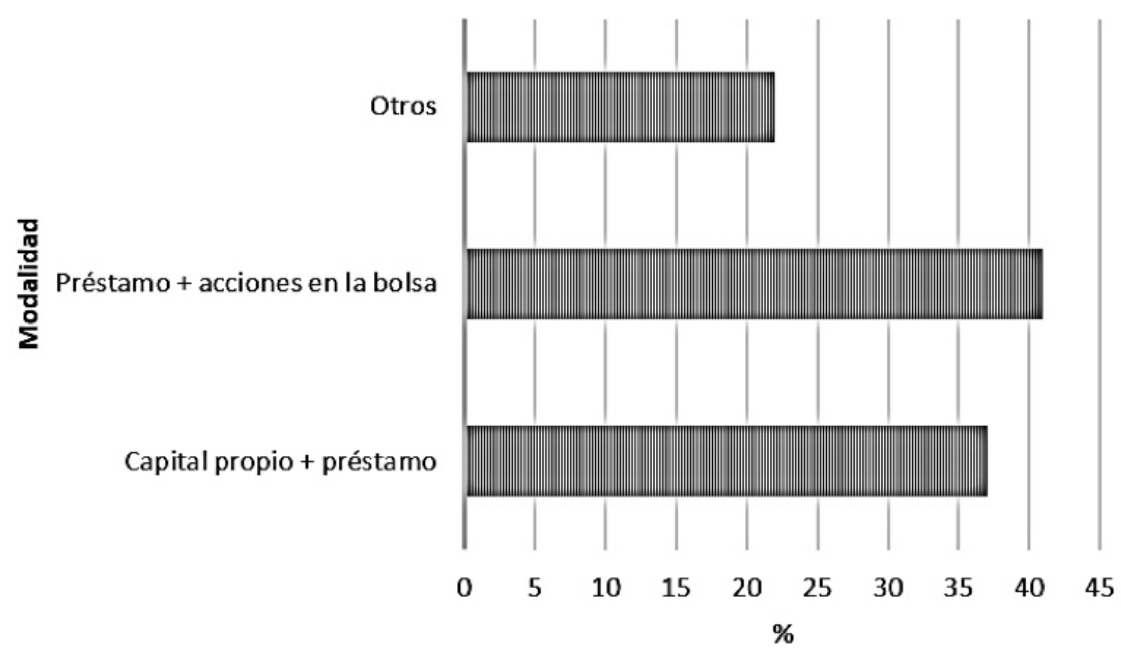

Figura 6. Fuentes de financiamiento de los procesadores de tomate de árbol (Solanum betaceum) en la zona centro de Ecuador. 2017.

Figure 6. Funding sources for tree tomato processors (Solanum betaceum) in Central Ecuador. 2017.

\section{Poscosecha: distribuidores}

\section{Hallazgos socioeconómicos}

Entre los distribuidores se identificó que el $50 \%$ eran medianas empresas y el $50 \%$ restante eran grandes empresas. Solo el $50 \%$ de los distribuidores eran miembros de asociaciones. El $50 \%$ poseía como socios estratégicos a cooperativas de crédito. Con respecto al financiamiento, el $72 \%$ de las empresas operaba con recursos propios y deuda, y el $28 \%$ dinamizaba su economía mediante apalancamiento bancario y acciones en la bolsa de valores.

\section{Hallazgos en la comercialización}

La comercialización de productos de tomate de árbol se ejecutaba por distribuidores mayoristas y minoristas. Los minoristas (retailers) canalizaban los productos hacia el consumidor final. Los mayoristas eran los encargados de la logística que implica la movilización de fruta hacia los centros distritales de distribución y lo centros de acopio para la exportación. Era habitual que previo a la entrega de pedidos se realizaran acuerdos verbales o mediante contratos escritos sobre aspectos relacionados a precio, periodos de suministro, condiciones de pago y entrega, entre otros. También se observó que el principal producto a base de tomate de árbol comercializado a nivel internacional era la pulpa procesada y congelado a $-20{ }^{\circ} \mathrm{C}$.

\section{Aspectos de mercado}

\section{Demanda}

En el año 2016 el precio promedio a nivel internacional de tomate de árbol aumentó en 4 \% con relación al 2015. Los valores promedio de este producto en los mercados de Estados Unidos, durante el año estudiado, 
registraron USD \$ 41,6/paquete de 30-42 unidades, que aproximadamente equivale entre USD \$ 5,5 y 6,8 $\mathrm{kg}^{-1} \mathrm{de}^{-}$ fruta. En contraste, el precio nacional de tomate de árbol a nivel productor en 2016 presentó una disminución de 5 $\%$ con relación al 2015. El precio promedio a nivel de productor para el año 2016 de tomate de árbol fue de USD $\$ 0,85 \mathrm{~kg}^{-1}$ (Figura 7).

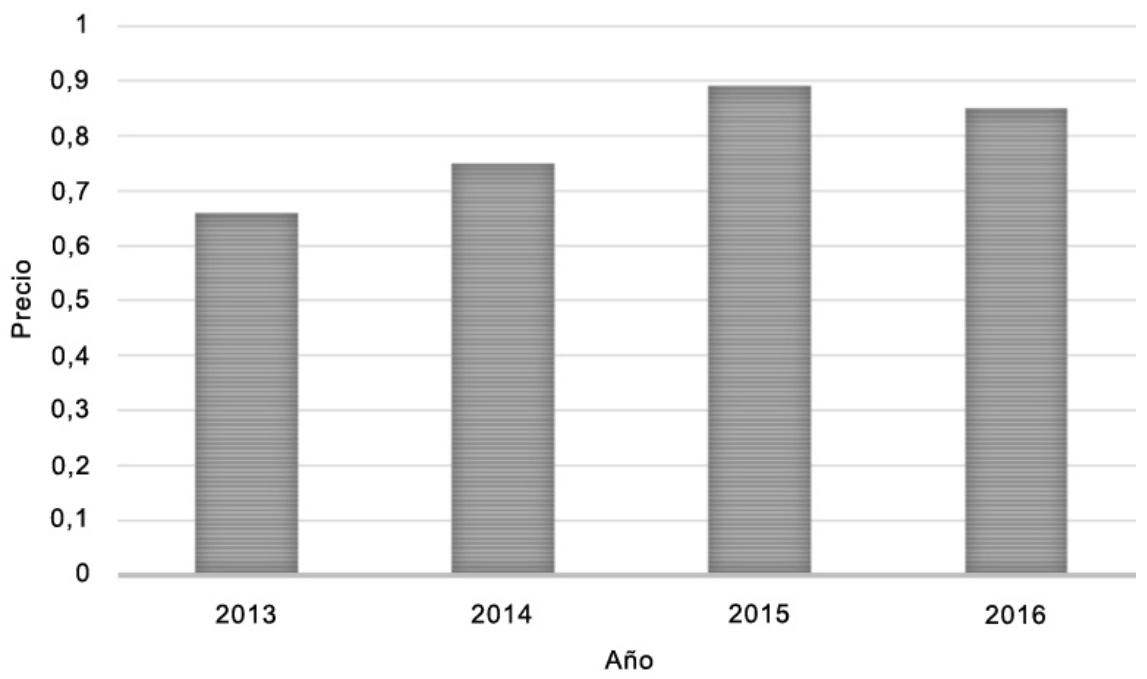

Figura 7. Precios nacionales para productor y mayoristas de tomate de árbol (Solanum betaceum) en la zona Centro de Ecuador, durante los años 2013-2016.

Figure 7. National prices for producers and wholesalers of tree tomatoes (Solanum betaceum) in Central Ecuador, during the years 2013-2016.

\section{Oferta}

Las exportaciones en el Ecuador durante el año 2016 registraron un incremento de $70 \%$. Dicho comportamiento refleja el incremento sostenido del comercio internacional del rubro mencionado, puesto que el año anterior existió un incremento del $13 \%$. En marzo de 2016, las exportación de tomate de árbol ecuatoriano registraron su mayor volumen, con 21000 t. Se observó que las entidades públicas competentes buscaban estructurar asociaciones destinadas a incrementar los volúmenes de producción de tomate con la visión de expandir el mercado internacional.

\section{Identificación de dinámica de gobernanza}

Se analizó la complejidad de la información compartida entre los actores de la cadena, la codificación de dicha información y el nivel de competencia del proveedor. Por tanto, según la clasificación propuesta por Frederick y Gereffi (2009), la dinámica de gobierno identificada fue la de mercado (Figura 8). El perfil identificado se caracterizó porque las transacciones eran repetitivas y fáciles de codificar. Los acuerdos y negocios se coordinaron mediante el mecanismo de precio al contado o contratos que contemplan periodos de pago cortos. Otro aspecto relevante fue que los costos transaccionales eran altos en relación con los que presentan cadenas con mayor dinamismo como frutilla, mora y papa (12 al $27 \%$ ). El porcentaje de estos costos en relación con el precio del productor estuvo entre el 32 y $58 \%$. Se identificó que los costos transaccionales sufrían un incremento debido al transporte, lo que evidencia aún fallos en la coordinación durante la logística y comercialización. 


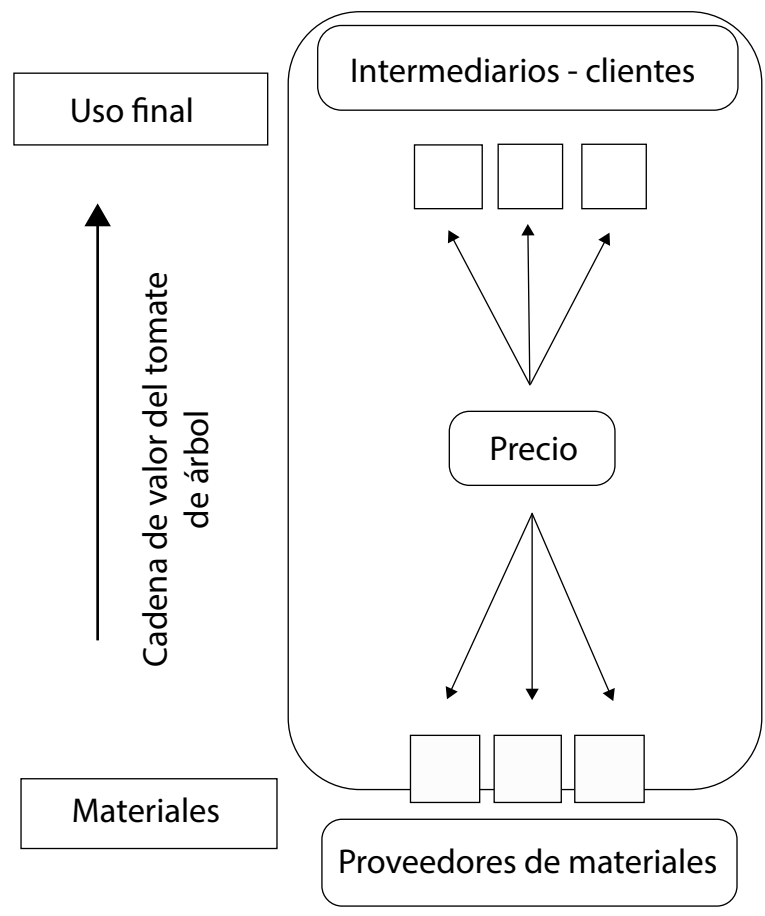

Figura 8. Dinámica de gobernanza de la red productiva de tomate de árbol (Solanum betaceum) en la zona centro de Ecuador. 2017.

Figure 8. Governance dynamics of the productive network of tree tomato (Solanum betaceum) in central Ecuador. 2017.

\section{Discusión}

La cadena de tomate de árbol es una cadena emergente, sus productores han preferido comercializar la fruta en mercados locales, ya que las transacciones han sido mayormente en efectivo y por tanto, logran un retorno seguro del capital invertido. Esta red se caracterizó porque el poder de negociación de sus productores fue relativamente alto, debido a que la demanda fue mayor a la oferta existente. Actualmente, la demanda ha experimentado un crecimiento, gracias a la diversidad de productos a base de tomate de árbol que ha generado la industria, como jugos, néctares, concentrados y salsas. Este aspecto diferencia a la red de tomate de las cadenas de mora, ajo y hortaliza en general. Otro aspecto interesante es la dinámica de comercio de la zona interandina, misma que ha sido considerada un importante nodo comercial hortofrutícola a nivel nacional, y que ha promovido la comercialización del tomate de árbol a través de sus centros de distribución distritales.

La etapa de producción ha poseído varias ventajas significativas de diversa índole, por ejemplo, la cosecha de la fruta ha sido durante todo el año, por lo que se ha podido obtener grandes volúmenes de producción (CORPOICA, 2009). Otra ventaja ha sido el aspecto financiero, según Villamarín et al. (2012), para la producción de un cultivo de 60 ha de tomate de árbol se ha requerido aproximadamente de USD \$ 8000 en comparación con otros cultivos similares que han requerido entre USD $\$ 12000$ y 15000 . El acceso a créditos ha sido una tercera ventaja, ya que los agricultores han registrado créditos de USD $\$ 17500$ en promedio, para la inversión en la producción. Además, 
Fernández (2018) indicó que el precio para el mercado doméstico del tomate de árbol fresco fue de USD \$ 0,85 por $\mathrm{kg}$, mismo que pudo variar hasta USD \$ 1,35 por kg; mientras que para el mercado internacional el precio del tomate de árbol fresco varió entre USD \$5,50 y 9,50 $\mathrm{kg}^{-1}$. Finalmente, la adaptabilidad del cultivo ha sido relevante, según Chañag-Miramag et al. (2017) hay veintinueve genotipos adaptados a las condiciones agroclimáticas ecuatorianas. Estos genotipos han sido comercializados en el mercado nacional e internacional. Colombia, el mayor productor de tomate de árbol a nivel mundial, cultivaba mayormente el genotipo colombiano anaranjado gigante y en menor proporción el genotipo morado gigante (Betancourth et al., 2003).

La etapa de poscosecha se caracterizó por presentar procesadores y comercializadores mayoristas y minoristas, quienes canalizaron la producción hacia mercados institucionales en cadenas hoteleras, restaurantes y servicios de catering (HORECA). Un estudio realizado en empresas del cantón Ambato, provincia de Tungurahua, afirmó que el 77,0 \% comercializaba tomate de árbol mínimamente procesado, y $23 \%$ con algún nivel de transformación. La situación en la industria alimentaria de Ecuador requiere de intervención pública para incentivar la diversificación de productos a base de tomate de árbol mediante oportunidades de mercado externo y créditos blandos. Ha existido una fuerte intervención de entidades públicas de salud durante todo el proceso de trasformación de la fruta, la cual ha sido ejecutada principalmente por la Agencia Nacional de Regulación, Control y Vigilancia Sanitaria (ARCSA).

\section{Conclusiones}

En la red productiva del tomate de árbol ecuatoriano se han involucrado instituciones públicas y privadas; estos actores han participado principalmente en la etapa de preproducción, dotando de recursos como semillas, fertilizantes, agroquímicos, capital, entre otros. Las actividades de soporte en esta etapa han sido el inicio del flujo de recursos tangibles necesarios para el desempeño de la red.

La etapa de producción ha contado con características sociodemográficas favorables. La intervención de participantes relativamente jóvenes cuya edad varía entre los 30 y 40 años se mostró clave para la sostenibilidad de la cadena. El costo estimado por kilogramo de producto fresco será de USD 0,85, y una hectárea de cultivo tenía una rentabilidad de al menos 18 \%; sin embargo, la cadena de tomate de árbol presentaba aspectos que requieren atención, como la formación técnica de productores en temáticas como poscosecha, logística de transporte, calidad alimentaria y el fortalecimiento de estructuras asociativas.

Las funciones de procesadores y distribuidores en la etapa de posproducción de la red estaban diferenciadas. Por un lado, las pequeñas empresas eran quienes participan en las actividades de valor agregado y pertenecían a asociaciones. Las empresas distribuidoras también presentaban un importante sentido de asociatividad, pero se caracterizaban por el modelo de negocios con el que operaban, por ejemplo, participan en la bolsa de valores, e implementan métodos de negociación de bajo crédito que generan flujos de efectivo positivos.

\section{Literatura citada}

Aguilar, D. 2017. Ecuador los problemas ambientales que deben resolverse en el 2017. MONGABAY, ECU. https://es.mongabay. com/2017/01/ecuador-los-problemas-ambientales-deben-resolverse-2017/ (consultado 12 nov. 2018).

Acosta, I. 2006. Balance del modelo agroexportador en América Latina al comenzar el siglo XXI. Mundo Agrar. 7(13). file:/// Users/pccmca/Downloads/844-Texto\%20de1\%20art\%C3\%ADculo-1630-1-10-20121105.pdf (consultado 14 nov. 2018.$).$

Arboleda, L., M. Duque, y J. Urrea. 2013. Significados del consumo de frutas y hortalizas en dos comunidades de zona rural del municipio de Turbo, Urabá Antioqueño. Saúde Sociedade 22:36-42. doi:10.1590/S0104-12902013000400025 
Barrera-Mosquera, V.I. de-los-Rios-Carmenado, E. Cruz-Collaguazo, and J. Coronel-Becerra. 2010. Analysis of available capitals in agricultural systems in rural communities: the case of Saraguro, Ecuador. Spanish J. Agric. Res. 8:1191-1207. doi:10.5424/sjar/2010084-1408

Barbier,E.2016.Sustainability and development.Ann.Rev.Res.Econ.8:261-280.doi:10.1146/annurev-resource-100815-095227

Betancourth, C., R. Goyes, y D. Bravo. 2003. Caracterización biológica de un virus en tomate de árbol (Solanum betaceum) presente en el departamento de Nariño. Tesis Lic., Universidad de Nariño, Nariño, COL.

Blandon, J., S. Henson, and J. Cranfield. 2009. Small-scale farmer participation in new afri-food supply chains: case of the supermarket supply chain for fruit and vegetables in Honduras. J. Int. Dev. 21:971-984. doi:10.1002/jid.1490

Brown, A., y P. Torres. 2012. La relación comercial Comunidad Andina- Unión Europea y la postura de Ecuador. Latinoamérica 55:75-99.

Cadilhon, J., P. Moustier, N. Poole, P. Giac-Tam, and A. Fearne. 2006. Traditional vs. modern food systems? Insights from vegetable supply chains to ho Chi Minh city (Vietnam). Dev. Policy Rev. 24:31-49. doi:10.1111/j.1467-7679.2006.00312.x

Campuzano, M. 2014. Greenhouse heating with geothermal energy: feasibility study for the Ecuadorian Alpine Grasslands (Páramo). Semantic Scholar, WA, USA. https://pdfs.semanticscholar.org/a79a/7b15a1783f4da12891f4a4662a24fd5fca17. pdf (accessed Oct. 30, 2018).

Carpio, J. 2018. Desarrollo local en los espacios rurales. Polis 2:14-28. doi:10.32735/S0718-6568/2002-N2-145

Castro, J. 2014. Fenología y crecimiento del tomate de árbol (Cyphomandra betacea) (Cav.) Sendt), cualtivado con coberturas plásticas en el suelo. Universidad de Nariño, COL. http://biblioteca.udenar.edu.co:8085/atenea/biblioteca/90244.pdf (consultado 10 jun. 2019).

CEPAL, FAO, e IICA. 2015. Perspectivas de la agricultura y del desarrollo rural en las Américas: una mirada hacia América Latina y el Caribe 2015-2016. CEPAL, Santiago, CHL. https://www.cepal.org/es/publicaciones/39023-perspectivas-laagricultura-desarrollo-rural-americas-mirada-america-latina (consultado 05 dic. 2018).

Chañag-Miramag, H.A., J. Viveros-Rojas, S. Álvarez-Ordoñez, H. Criollo-Escobar, y L.E. Lagos-Mora. 2017. Evaluación de genotipos de tomate de árbol (Cyphomandra betacea (Cav.) Sendt.] frente al ataque de Phytophthora infestans (Mont.) de bary sensu lato. Rev. Colomb. Cienc. Hort. 11:11-20. doi:10.17584/rcch.2017v11i1.4725

CORPOICA (Corporación Colombiana de Investigación Agropecuaria). 2009. Manual de manejo cosecha y poscosecha de tomate de árbol. CORPOICA, Bogota, COL.

Coyle, J.J., C.J. Langley, R.A. Novack, and B.J. Gibson. 2016. Supply chain management: a logistics perspective. Cengage Learning, Mason, AUT. https://cengage.com.au/product/division/university/title/supply-chain-management-a-logisticsperspecti/isbn/9781305859975 (accessed Oct. 30, 2018).

Craig, R., P. Carter, and E. Liane. 2011. “Sustainable supply chain management: evolution and future directions”. Int. J. Physical Distr. Logistics Manag. 41:46-62. doi:10.1108/09600031111101420

Demenus, W., and P. Crespo. 2011. Productive chains and rural economic development in Latin America. Consorcio de Consejos Nacionales de Ecuador, Quito, ECU. http://www.congope.gob.ec/wp-content/uploads/2014/08/Cadenas-Productivas-yDesarrollo-Economico-Rural-en-Latinoamerica.pdf (consultado 20 nov 2018).

FAO. s.f. Mejoramiento de la calidad e inocuidad de las frutas y hortalizas frescas: bajo un enfoque práctico. FAO, Roma, ITA. http://www.fao.org/ag/agn/pfl_report_en/_annexes/Annex4/Ecuador/Importancesummary.xls (consultado 23 nov. 2018).

FAO. 2011. Pérdidas y desperdicios de alimentos en el mundo. FAO, Roma, ITA. http://www.fao.org/3/a-i2697s.pdf (consultado 02 dic. 2018). 
FAO. 2018. Frutales andinos: tomate de árbol. FAO, Roma, ITA. http://www.fao.org/tempref/GI/Reserved/FTP_FaoRlc/old/ prior/segalim/prodalim/prodveg/cdrom/contenido/libro10/cap03_4.htm (consultado 01 sep. 2019).

Feican, C. 2016. Descripción agronómica del cultivo de tomate de árbol. Rev. Agrocienc. 9:78-86. doi:10.13140/ RG.2.2.18036.35208

Fernández, D. 2018. Plan de negocios para la comercialización de tomates de árbol orgánicos en almíbar hacia Alemania. Universidad de Las Américas, ECU. http://dspace.udla.edu.ec/bitstream/33000/8585/1/UDLA-EC-TINI-2018-03.pdf (consultado 12 dic. 2018).

Flores, P. 2007. Tomate de árbol. Escuela Politécnica del Ejército, ECU. https://repositorio.espe.edu.ec/bitstream/21000/2311/2/ T-ESPE-017207.pdf (consultado 02 may. 2019).

Frederick, S., and G. Gereffi. 2009. Value chain governance: Briefing paper. Market Links, USA. https://www.marketlinks.org/ sites/marketlinks.org/files/resource/files/vc_governance_briefing_paper.pdf (accessed Nov.10, 2018).

Gayá, R., y K. Michalczewsky. 2014. El comercio intrarregional sudamericano: Patrón exportador y flujos intraindustriales. IADB Improving Life Publications, USA. https://publications.iadb.org/es/publicacion/13755/el-comercio-intrarregionalsudamericano-patron-exportador-y-flujos (consultado 10 nov 2018).

Gereffi, G. 1994. The organization of buyer-driven global commodity chains: How US retailers shape overseas production networks. In: G. Gereffi and M. Korzeniewicz, editors, Commodity chains and global capitalism. Praeger, Westport, CT, USA. p. 95-122. Duke University, NC, USA. https://dukespace.lib.duke.edu/dspace/bitstream/handle/10161/11457/1994_ Gereffi_Role\%20of\%20big\%20buyers\%20in\%20GCCs_chapter\%205\%20in\%20CC\&GC.pdf?sequence=1 (accessed Nov. 5, 2018).

Giacobone, G., L. Castronuovo., V. Tiscornia, y L. Allemandi. 2018. Análisis de la cadena de suministro de frutas y verduras en Argentina. Fundación Inter Americana del Corazón (FICA), ARG. https:/www.ficargentina.org/documentos/analisis-dela-cadena-de-suministro-de-frutas-y-verduras-en-la-argentina-2018/ (consultado 15 dic. 2018).

Gil-Méndez, J. 2015. Neoliberalismo, políticas agrarias y migración. consecuencias de un modelo contra los productores. Ra Ximhai 11:145-162.

Glas, J., V. Alvarado, S. León, y J.C. Parra. 2015. Política Industrial del Ecuador 2016-2025 más industrias mayor desarrollo. Ministerio de Industrias y Productividad, Ministerio Coordinador de Producción, Empleo y Competitividad, ECU. https:// issuu.com/dircommipro/docs/politicaindustrialweb_16-dic-16ok (consultdo 23 nov. 2018).

Gunsha, C. 2016. Costos de producción del cultivo de frutilla. Universidad Nacional de Chimborazo, Riobamba, ECU. http:// dspace.unach.edu.ec/bitstream/51000/2789/1/UNACH-FCP-ECO-2016-0014.pdf (consultado 16 sep. 2019)

Hawkes, C., and M. Ruel. 2006. The links between agriculture and health: An intersectoral opportunity to improve the health and livelihoods of the poor. Bull. World Health Organ. 84:985-991. doi:10.1590/S0042-96862006001200015

Herforth, N., L. Theuvsen., W. Vásquez, and M. Wollni. 2015. Understanding participation in modern supply chains under a social network perspective: Evidence from blackberry farmers in the Ecuadorian Andes. AgEcon Search. https:// ageconsearch.umn.edu/record/197709/files/GlobalFood_DP57.pdf (accessed Oct. 20, 2018).

León, J., P. Viteri, y G. Cevallos. 2004. Manual del cultivo de tomate de árbol. INIAP, ECU. http://repositorio.iniap.gob.ec/ handle/41000/827 (consultado 20 jul. 2019).

López, A. 2015. Cambio climático y actividades agropecuarias en América Latina. CEPAL, Santiago, CHL. https://repositorio. cepal.org/bitstream/handle/11362/39824/1/S1501286_es.pdf (consultado 02 sep. 2019).

MAG (Ministerio de Agricultura y Ganadería). 2018. Boletín situacional: tomate de árbol. FLIPHTML5, USA. http://fliphtml5. com/ijia/efww/basic (consultado 10 dic. 2018). 
MAGAP (Ministerio de Agricultura, Ganaderia, Acuacultura y Pesca). 2016. La política agropecuaria ecuatoriana. Hacia el desarrollo territorial rural sostenible 2015-2025. I Parte. MAGAP, Quito, ECU. http://servicios.agricultura.gob.ec/ politicas/La\%20Pol\%C3\%ADticas\%20Agropecuarias\%20al\%20\%202025\%20I\%20parte.pdf (consultdo 15 dic. 2018 ).

Melo, C., and G. Hollander. 2013. Unsustainable development: Alternative food networks and the Ecuadorian Federation of Cocoa Producers, 1995-2010. J. Rural Stud. 32:251-263. doi:10.1016/j.jrurstud.2013.07.004

MIPRO (Ministerio de Industrias y Productividad). 2018. Catálogo de productos nacionales. Directorio de Mipymes: grandes empresas y artesanos 2018 - 2019. Ministerio de Industrias y Productividad, Quito, ECU. https://www.industrias.gob. ec/13573-2/ (consultado 15 jun. 2019).

Miranda, D. 2011. Estado actual de fruticultura colombiana y perspectivas para su desarrollo. Rev. Bras. Frutic. 33:199-205. doi:10.1590/S0100-29452011000500023

Olaizola, C., M. Esté, M. Tapia, A. Carmona, and U. Emaldi. 2006. Towards a program to promote fruit and vegetable consumption in Venezuela. Rev. Chilena Nutr. 33:18-29. doi:10.4067/S0717-75182006000300015

Ordoñez, D. 2012. El comercio exterior del Ecuador: Análisis del intercambio de bienes desde la colonia hasta la actualidad. Servicios Académicos Intercontinentales S.L., ESP. http://www.eumed.net/cursecon/ecolat/ec/2012/ddoi.pdf (consultado 04 dic. 2018).

Orjuela, J.A., C.A. Castañeda, y M.E. Calderón. 2008. Análisis de la cadena de valor en las estructuras productivas de uvilla y tomate de árbol en la Provincia de Sumapaz y el Distrito Capital. Rev. Cient. Tecnol. Fac. Ing. 13:4-12.

Padilla, W., and J. García. 2018. Data association methodology to improve spatial predictions in alternative marketing circuits in Ecuador. Comput. Intell. Neurosci. 2018:6587049. doi:10.1155/2018/6587049

Páez, C. 2018. El régimen impositivo a la propiedad agrícola en el Ecuador y los perjuicios socioeconómicos que causó su existencia. Universidad Central del Ecuador, Quito, ECU. http://www.dspace.uce.edu.ec/bitstream/25000/16600/1/TUCE-0013-JUR-068.pdf (consultado 01 ene. 2019).

Pérez, E. 2004. El mundo rural latinoamericano y la nueva ruralidad. Nómadas 20:180-193.

Pérez, M. 2011. Los costos de producción de mora y el nivel de ingresos de los agricultores. Universidad Técnica de Ambato, Ambato, ECU. http://repo.uta.edu.ec/bitstream/123456789/1449/1/TE0007.pdf (consultado 17 sep. 2019)

PROECUADOR (Instituto de Promoción de Exportaciones e Inversiones). 2016. Boletín mensual de comercio exterior. Diciembre 2015 - enero 2016. ISSUU, Quito, ECU. https://issuu.com/pro-ecuador/docs/boletindic_eneh (consultado 10 dic. 2018).

Quiroso, O., L. Temple, R. Alves, J. Ruales, and H. Guzman. 2008. Producing added value from underutilized tropical fruit crops with high commercial potential (PAVUC). CIRAD, FRA. http://agritrop.cirad.fr/560363/ (accessed Nov. 10, 2018).

Reardon, T., and L. Flores. 2006. Viewpoint: "Customized competitiveness" strategies for horticultural exporters - Central America focus with lessons from and for other regions. Food Policy 31:483-503. doi:10.1016/j.foodpol.2006.05.001

RIMISP (Latin American Institute of Rural Development). 2019. Grupo de diálogo andino: su aporte a la agricultura familiar sostenible. RIMISP, ECU. https://rimisp.org/noticia/grupo-de-dialogo-andino-dos-anos-aportando-a-una-agriculturafamiliar-sostenible/ (consultado 15 nov. 2018).

Rodríguez, E. 2015. La crisis del sector agropecuario colombiano ¿Cuál es la responsabilidad de las políticas públicas? Rev. Fac. Cienc. Econ. Adm. 16:159-174. doi:10.22267/rtend.151601.38

Sánchez, J. 2017. Mercado de productos agrícolas ecológicos en Colombia. Suma Neg. 8:156-163. doi:10.1016/j. sumneg.2017.10.001 
Scott, G.J. 2014. Agregando valores a las cadenas de valor. RAE 54:67-79. doi:10.1590/S0034-759020140107

Sukhatme, P.V. 1946. Bias in the use of small-size plots in sample surveys for yield. Natur. 157:630. doi:10.1038/157630b0

Urquilla, A. 2012. La exportación de alimentos a los Estados Unidos: principales desafíos para el sector agroindustria. Universidad Francisco Gavidia, ESV. http://ri.ufg.edu.sv/jspui/bitstream/11592/8313/1/La\%20exportación\%20de\%20 alimentos\%20a\%20los\%20Estados\%20Unidos\%3a\%20principales\%20desaf\%C3\%ADos\%20para\%20el\%20sector\%20 agroindustria.pdf (consultado 10 dic. 2018).

Vergara, W., A. Ríos., P. Trapido, y H. Malarín. 2014. Agricultura y clima: Futuro en América Latina y el Caribe. BID, NY, USA. https://publications.iadb.org/es/publicacion/16673/agricultura-y-clima-futuro-en-america-latina-y-el-caribe-impactossistemicos-y (consultado 5 may. 2019).

Villamarín, M., I. Muñoz, y C.A. Benavides. 2012. Respuesta del tomate de árbol (Cyphomandra betacea (Cav.) Sendt) a la fertilizacióncon N-P-K en el suelo de la vereda pradera bajo Pasto. Universidad de Nariño, COL. http://sired.udenar.edu. co/4061/1/86169.pdf (consultado 10 ene. 2019).

Viteri, P., J. León, W. Vásquez, C. Encalada, A. Martínez, J. Revelo, M. Poso, y M. Hinojosa. 2010. Solanáceas silvestres utilizadas como portainjertos de tomate de árbol (Solanum betaceum Cav.) con alto rendimiento, resistencia a enfermedades y mayor longevidad. Instituto Nacional de Investigaciones Agropecuarias - INIAP, ECU. http://repositorio. iniap.gob.ec/bitstream/41000/5137/1/iniapscbd371.pdf (consultado 01 sep. 2019).

Wendt, J., and J. Izquierdo. 2003. Management of appropriate agricultural biotechnology for small producers: case study Ecuador. Electon. J. Biotechnol. 6(1):3-7. doi:10.2225/vol6-issue1-fulltext-i02pdf

Wendt, J., and J. Izquierdo. 2003. Management of appropriate agricultural biotechnology for small producers: case study Ecuador. Electon. J. Biotechnol. 6:15-16. http://www.ejbiotechnology.cl/index.php/ejbiotechnology/article/view/v6n1i02pdf/561 (accessed Nov.18, 2018). 